\title{
Communication et implantation bureautique dansle franchisage de diffusion
}

André A. Lafrance, Daniel Girard, Hélène Akzam et Sylvie Lavoie

\section{(2) OpenEdition}

12 Journals

Édition électronique

URL : http://journals.openedition.org/communicationorganisation/1578

DOI : 10.4000/communicationorganisation. 1578

ISSN : $1775-3546$

Éditeur

Presses universitaires de Bordeaux

Édition imprimée

Date de publication : 1 novembre 1992

ISSN : 1168-5549

\section{Référence électronique}

André A. Lafrance, Daniel Girard, Hélène Akzam et Sylvie Lavoie, "Communication et implantation bureautique dansle franchisage de diffusion », Communication et organisation [En ligne], 2 | 1992, mis en ligne le 26 mars 2012, consulté le 20 avril 2019. URL : http://journals.openedition.org/ communicationorganisation/1578 ; DOI : 10.4000/communicationorganisation.1578

Ce document a été généré automatiquement le 20 avril 2019

(c) Presses universitaires de Bordeaux 


\title{
Communication et implantation bureautique dansle franchisage de diffusion
}

\author{
André A. Lafrance, Daniel Girard, Hélène Akzam et Sylvie Lavoie
}

1 Deux phénomènes, tout aussi marquants, retiennent l'attention des observateurs de la scène organisationnelle. Il y a, d'un côté, le développement des réseaux d'entreprises franchisées qui a grandement modifié le portrait traditionnel de la vente de produits et de services. Le succès de cette modalité commerciale s'expliquerait par les avantages que toutes les parties semblent en tirer. Le franchiseur étend ses activités sans avoir à y consacrer de lourds investissements. Le franchisé devient son propre patron tout en profitant de l'expertise de quelqu'un d'autre. Le consommateur, quant à lui, y trouve, à la fois, le service "personnalisé » par la "présence» du propriétaire de la franchise et la sécurité d'un produit ou d'un modèle commercial s'appuyant sur la crédibilité du franchiseur ou la promotion du nom corporatif qui lui est associé.

D'un autre côté, on assiste à l'informatisation « galopante » des activités de gestion avec les difficultés inhérentes à toute opération qui remet en question les pratiques de l'organisation. L'implantation bureautique transgresse les frontières du statu quo l'«homéostasie » téléologique de l'analyse systémique) non pas tellement parce que le logiciel force ses utilisateurs à «penser » et « gérer » comme ceux qui l'ont conçu (cela est, certes, troublant), mais surtout parce qu'il rend plus apparents les marges de manœuvre et les mécanismes de prisea de décision des utilisateurs. Il n'y a plus moyen de cacher, derrière une ambiguïté faisant l'affaire des parties impliquées, les écarts conceptuels et opérationnels dont témoignent leurs pratiques respectives.

3 Le gestionnaire d'un réseau d'entreprises franchisées se trouve confronté à une tâche apparemment condamnée à l'échec : réaliser une implantation bureautique qui permettra à ses franchisés de se maintenir face aux organisations polycentriques (réseaux) ou monocentriques (filiales ou succursales) concurrentes sans, pour autant, les amener à 
prendre conscience de la concurrence, apparente ou réelle, existant entre leurs intérêts et ceux du franchiseur.

4 Le succès de cette implantation bureautique dépendra des structures communicationnelles soutenant le fonctionnement quotidien du réseau. Pour l'analyser, il faut définir la relation franchiseur-franchisés, d'abord dans sa compréhension contractuelle, puis dans son extension communicationnelle. Est-il possible d'induire de la pratique des principaux réseaux d'entreprises franchisées une identification précise des spécificités d'une relation franchiseur-franchisés? Comment circonscrire les paramètres communicationnels propres au franchisage afin de bâtir une stratégie facilitant le travail des franchiseurs « en voie de développement informatique »?

\section{La compréhension contractuelle}

Il semble donc, à première vue, assez simple de décrire les mécanismes du franchisage. C'est un système de commercialisation caractérisé par une entente contractuelle verticale entre une entreprise maitresse (un franchiseur) et des subordonnés (des franchisés). « La relation entre les deux parties est comparable à celle d'un employeur vis-à-vis de ses employés » (Rubin, 1978).

6 On y trouve une entreprise initiatrice d'un savoir-faire (un franchiseur) qui permet à d'autres (des franchisés) d'utiliser ce savoir-faire (préalablement expérimenté) en échange d'un montant initial et de rémunérations sous forme de redevances.

7 Afin que chacune des deux parties en tire des avantages, le contrat qui les relie se définit par une entente sur le degré de dépendance réciproque entre franchiseur et franchisés, (variant selon le type de franchise dont il est question), une zone territoriale délimitée pour chacun des franchisés et une durée variable.

8 Les deux parties se rattachent au même signe d'adhésion défini par le franchiseur, offrant aux consommateurs, des produits et/ou des services identifiés à ce signe d'adhésion.

Dans ces éléments de définition, on peut déjà observer qu'il ne s'agit pas d'une méthode de collaboration ou de coopération égalitaire puisque les deux parties ne sont pas sur le même pied. Le franchiseur détermine les règles du jeu.

10 Si les auteurs de contrats ou d'analyses théoriques sont parfois victimes de glissements sémantiques quant à la compréhension et à l'utilisation du terme "franchises", ils semblent s'accorder sur les sortes de franchises autant qu'ils s'en tiennent à leur vocation commerciale ou industrielle. Nous avons ramené les classifications proposées à une dualité : le franchisage de reproduction et le franchisage de diffusion.

\section{Le franchisage de reproduction}

11 Le franchisé « de reproduction ou de duplication » ne fait que reproduire, dans sa propre entreprise, l'activité du franchiseur. Pour cela il bénéficie d'un système de référence à un style particulier de service et/ou à des moyens de fabrication définis par le franchiseur. Ce transfert entre franchiseur et franchisés s'effectue non seulement au niveau du savoirfaire proprement dit, mais aussi au niveau du processus de fabrication. On y trouve le franchisage industriel et le franchisage de service. 
12 Dans le franchisage industriel, on parle alors de lifreding (Licensing, Franchising, Export and Direct Investment), ce qui consiste, pour une entreprise qui a réussi sur son marché, à rentabiliser ses investissements initiaux en vendant un "package » technique global (fabrication, marketing, gestion) capable d'assurer le succès d'exploitation pour des entreprises aptes à recevoir ce "package » et à l'adapter localement» (De Mendez et Lehnish, 1984).

13 Le « lifreding » est donc un système de "franchise totale » permettant à des entreprises industrielles d'offrir un "package» de performances adaptées à l'exportation dans chacun des pays visés et d'assurer ainsi à la marque une couverture géographique remarquable et rapide. Les compagnies Yoplait et Coca-Cola sont de bons exemples d'entreprises industrielles qui ont franchisé leurs implantations internationales.

14 Après le franchisage industriel, on trouve le franchisage de service qui est essentiellement la reproduction d'un savoir-faire. Le franchisé reproduit et vend des services préalablement créés et définis par un franchiseur. On en dénote deux catégories. Les franchises de services de type tertiaire pur et celles impliquant un investissement assez lourd, qui les rapproche des franchises industrielles, comme les franchises de type hôtelier.

15 Le franchisage dit " de modèle tertiaire pur ", est fondé sur une conception particulière d'un service qui convient aux besoins d'un segment de clientèle bien déterminé. C'est l'originalité du service qui le fait vendre. Par exemple, la chaîne de location d'automobiles Hertz fut la première à proposer à ses clients de laisser la voiture dans une autre ville que celle où ils l'avaient louée.

16 Le franchisage dit «de type hôtelier » (incluant les chaînes d'hôtels ou de motels; les chaînes de restaurants, snacks-bars et fast-food ; les chaînes de cafés-bars ; les chaînes de clubs sportifs ; etc.) est caractérisé par la facilité de l'enseignement des prestations qu'elle offre. Elle implique néanmoins un choix de la clientèle-cible ainsi qu'une identification de ses besoins. «Ce choix se fait en fonction de la catégorie de l'établissement projeté, de la mobilité de cette clientèle qui doit pouvoir retrouver les établissements franchisés lors de ses déplacements et de l'identité typologique de cette clientèle dans les régions où l'on veut développer la chaîne » (Chambre Nationale des conseillers financiers, 1975).

17 Par contre, la contrainte principale se situe au niveau de l'investissement qui est relativement très lourd. Les deux parties, soit le franchiseur et le franchisé, doivent immobiliser d'importantes sommes d'argent. De plus, le nombre de ces établissements doit être assez important pour que le franchiseur puisse tirer une rentabilité valable de son investissement initial. «On peut donc dire que le franchisage de type hôtelier est multinational à la fois par sa nature (effet de châne) et par nécessité (exiguïté des territoires nationaux)»(Chambre Nationale des conseillers financiers, 1975). Les restaurants McDonald en sont un très bon exemple.

18 Nous allons, quant à nous, nous intéresser à l'autre type de franchisage, celui dit « de diffusion ». C'est dans ce type que se déroulent les expériences les plus intéressantes d'implantation bureautique associée à la gestion.

\section{Le franchisage de diffusion}

19 Les franchisés "de diffusion » se concentrent sur le commerce de détail. Le franchiseur doit les approvisionner, en partie ou en totalité, en marchandises ; il est tenu de fabriquer 
ou de choisir les produits qui feront partie de sa centrale d'achat. La préoccupation majeure du franchiseur est de distribuer des produits qu'il a fabriqués ou sélectionnés, par l'entremise d'un réseau de détaillants. Ainsi il s'assure, par le biais de sa centrale d'achat, d'une certaine maîtrise sur le canal de distribution et d'une certaine uniformité parmi les franchisés. (Nègre, 1988)

Dans le franchisage de diffusion, on retrouve un modèle pur et des modèles associés de façon prioritaire à la production et à l'exploitation.

Dans le franchisage dit de "diffusion - modèle pur ", le franchiseur ne produit pas luimême les produits. Il les achète ou les fait fabriquer par un ou des fournisseurs indépendants et les revend par la suite aux franchisés. Ceux-ci bénéficient donc du pouvoir d'achat du franchiseur. Le franchiseur joue, somme toute, un rôle de distributeur. L'activité du franchiseur et des franchisés étant relativement semblable, les relations entre ces deux entités risquent d'être plus équilibrées que dans la franchise de production. (Clément, 1981)

Dans le cas du franchisage dit de " diffusion-production », le franchiseur manufacture luimême les produits qu'il vend aux franchisés. Il est un industriel qui désire, par le franchisage, contrôler la distribution de ses produits. Le contrat, unissant franchiseur et franchisés, met l'accent sur un ou plusieurs produits particuliers. Car le franchiseur, dans la plupart des cas, ne peut produire la gamme complète des produits qui seront vendus par le franchisé. Le franchiseur s'intéressant d'abord à la production et le franchisé à la vente, leurs activités respectives sont de nature différente. Les rôles sont répartis. Mais le franchiseur risque de dominer les relations qui s'établiront entre lui et les franchisés (Clément, 1981).

Ce qui caractérise le franchisage dit de " diffusion-exploitation » ou " clé en main », c'est le contrôle que le franchiseur exerce sur ses franchisés. Il lui accorde le droit et la permission d'exploiter une entreprise conformément à une formule où tout a été pensé selon des modes d'opération précis et des procédures contrôlées par le franchiseur. Le franchisé utilise de façon exhaustive le savoir-faire, l'expertise, la marque de commerce ou autres noms distincts du franchiseur, les modes d'identification tels la forme particulière du bâtiment, des couleurs, des équipements particuliers ainsi que tous les autres éléments qui identifient la formule du franchiseur.

Le "franchise package ", l'essence même de l'opération "clés en main », est l'ensemble des « ingrédients de la recette » qui permettra au franchisé d'opérer son commerce avec succès, s'il respecte les directives du manuel d'opération.

D'ailleurs, le plan d'organisation de l'entreprise du franchisé est presque entièrement développé par le franchiseur. "Ce plan intègre même le contrôle exercé par le franchiseur, non seulement sur la bannière du franchisé, mais aussi sur l'ensemble de ses activités » (Levasseur et associés, 1983). En fait la société de franchisage a le monopole des approvisionnements. Le franchiseur contrôle les procédures d'opération et d'exploitation en ce qui concerne la constitution et le développement des registres, des contrôles financiers, de la publicité et des méthodes de mise en marché. Il prend en main tous les aspects techniques de la gestion du personnel, forme le franchisé et ses employés, établit les échelles de salaires, les programmes d'avantages sociaux, fait les paies et effectue les remises aux gouvernements. En fait il gère tout, de l'idée même du concept jusqu'à la couleur des murs et des tapis qu'il veut uniforme à l'intérieur de chacune de ses 
franchises. Il ne reste plus au franchisé qu'à ouvrir ce qui sera, dorénavant, son magasin et de veiller à sa gestion quotidienne.

\section{Les échanges entre le franchiseur et les franchisés}

Quel que soit le modèle de franchisage, il y a, dans son extension communicationnelle, deux éléments d'échange : ce qui est vendu au franchisé et ce qui est payé au franchiseur. Ces éléments varient d'un réseau à l'autre et, surtout, ils évoluent à l'intérieur d'un même réseau, tenant compte des perceptions fluctuantes de chacune des parties (communication interne au réseau) et des conditions changeantes du marché dans lequel elles opèrent (communication externe).

\section{Ce qui est vendu}

L'entreprise franchisée s'associe au franchiseur dans une relation plus ou moins englobante. Nous allons identifier quatre éléments du courant franchiseur-franchisés. Ils représentent les grandes tendances actuelles en ce domaine. Mais il faut signaler que l'imagination de certains franchiseurs dépasse toute tentative d'identification et même de raisonnement!

\section{La bannière}

La bannière offre au franchisé un soutien promotionnel sous la forme d'un nom jouissant d'une valeur de reconnaissance positive au sein de la clientèle visée et/ou d'instruments publicitaires collectifs. Si le nom n'a qu'une valeur relative à l'effort commun projeté, la production des instruments publicitaires amène le réseau à ressembler davantage à un regroupement de marchands dont les membres feraient affaire avec un service professionnel de publicité ou de marketing. Alors le pouvoir de décision a tendance à se déplacer du franchiseur aux franchisés qui peuvent toujours, collectivement, aller chercher ce service ailleurs que chez le franchiseur (« fausses franchises »).

La force de négociation du franchiseur repose donc sur la valeur du nom qui soutient la bannière. C'est pourquoi certains franchiseurs ont d'abord investi des sommes importantes dans la promotion d'un nom qu'ils espéraient, par la suite, rentabiliser en vendant des franchises de bannière. On assiste au marketing d'un nom avant même qu'il puisse se concrétiser dans des entreprises offrant les produits ou les services qui y sont liés. Plusieurs ont tenté de court-circuiter cette exigence en " achetant » et en associant à leur bannière un nom jouissant d'une certaine notoriété comme celui d'une vedette de sport ou de spectacle.

\section{Le produit}

Il est certain que toute franchise est associée à un produit, c'est-à-dire qu'elle opère dans un domaine spécialisé d'activités commerciales.

31 C'est la possibilité, pour le franchisé, d'offrir un ou des produits particuliers à un prix et dans un territoire fixés par le franchiseur qui en a le monopole de la distribution ou de la 
fabrication. C'est une forme de licence sans laquelle le franchisé ne pourrait obtenir ce produit et l'offrir à sa clientèle. Par exemple, les entreprises œuvrant dans l'innovation technologique (de l'automobile à l'ordinateur) ont toujours été fortement tentées de contrôler les conditions de vente de leurs produits en franchisant ceux à qui elles voulaient confier cette tâche sans pour autant se faire accuser, par les gouvernements ou les revendeurs, de pratiquer un monopole vertical handicapant les « lois du marché ». Ce type de franchise peut aussi permettre à un producteur d'équilibrer sa mise-en-marché en différenciant ses produits au moyen de plusieurs réseaux de franchises (Lee, 1984).

Signalons néanmoins que si le contrat de franchisage contient des éléments de concession, il ne peut se ramener à un contrat de concession. En effet, ce dernier ne comporte pas, comme le contrat de franchisage, de portée contraignante, de délimitation précise des droits et obligations de chacune des parties, autres que ce qui concerne les produits ou services faisant office de concept original (Chambre Nationale des Conseillers Financiers, 1975).

Il faut aussi ajouter que le produit pourrait ressembler à une bannière lorsqu'il se transforme en locomotive pour un ensemble de produits associés plus ou moins artificiellement à ce dernier (Stem, 1985 ; Tauber, 1981).

\section{La centrale d'achat}

Si le franchiseur n'est pas un producteur, mais un distributeur, il offre à ses franchisés les services d'une centrale d'achat.

L'initiative d'un tel regroupement peut même, dans certains cas, venir des points de vente. Ceux-ci se donnent un centre de coordination de leurs achats collectifs afin de bénéficier des économies de volume dont jouissent déjà leurs plus gros concurrents. Ces coopératives ou associations de marchands-membres se sont parfois transformées en véritables franchiseurs offrant leurs services d'achats et de distribution à des nonmembres.

Mais ce sont surtout des distributeurs qui ont cherché à établir et maintenir des liens plus étroits avec leurs propres clients. Ils créent parfois une seconde entité légale, le franchiseur, qui fait affaire avec le distributeur au nom de ses franchisés, sans pour autant rejeter officiellement les autres clients qui souhaitent maintenir leur autonomie. Dans ces cas, le plus difficile pour le nouveau franchiseur, c'est de réaliser le passage d'une entreprise centrée sur sa propre performance à une entreprise consacrée à l'amélioration de la performance de ses franchisés (Edwards, 1987).

L'argument initial est donc la mise en commun ou la remise à un tiers des achats de l'entreprise afin d'avoir accès aux avantages de volume et de force de négociation face aux fabricants. Là encore, on y associe habituellement une bannière afin de combiner les deux arguments (promotion et économie) et de renforcer le sentiment d'appartenance des franchisés. Si les économies de volume peuvent être, à tout moment, contestées par le franchisé ${ }^{1}$ ou concurrencées par un autre franchiseur, la bannière est une confirmation visible et un rappel incontestable du lien de franchise. 


\section{Le savoir-faire}

d'existence, les nouvelles entreprises franchisées ne connaissaient, au Canada, que $10 \%$ d'échecs en comparaison avec $70 \%$ pour les autres entreprises. Cela est probablement dû non seulement à la formation donnée par les franchiseurs et à l'encadrement plus serré des franchisés, mais, aussi, à la sélection des franchisés par des franchiseurs soucieux de ne pas confier leur image/produit à des partenaires trop maladroits ou trop mal financés pour absorber leurs premières maladresses! D'ailleurs " l'absence d'échec » ne semble pas être une garantie de succès puisqu'une étude sur le taux de succès des entreprises américaines œuvrant dans le domaine de la teinturerie n'a pu démontrer aucune différence entre celles qui étaient franchisées et celles qui ne l'étaient pas. Dans les deux cas, c'étaient plutôt l'âge et la grosseur des entreprises qui offraient les corrélations les plus intéressantes avec l'objet de l'étude (Bracker et Pearson, 1988).

\section{Les critères de séduction}

Quel que soit le type de franchise, il est important de rappeler que la relation franchiseurfranchisé est caractérisée par les éléments d'un échange commercial.

Le franchisé a un besoin que le franchiseur prétend pouvoir satisfaire. Contrairement à la formule classique de création d'entreprise, il y a, en fait, peu de franchisés potentiels qui déterminent d'abord un besoin "environnemental » qu'ils chercheraient, ensuite, à satisfaire en utilisant un modèle de franchise approprié. Il s'agit plutôt d'un marché où l'offre va au devant de la demande en créant un modèle qui cherche à s'imposer par son originalité, son efficacité ou sa popularité. C'est l'un des cas décrits par l'humoriste comme « une solution à la recherche d'un problème ».

Nous venons de mentionner les trois critères de séduction présentés par un modèle de franchise.

1 - L'originalité, c'est la recette innovatrice dont les ingrédients sont jalousement conservés et contrôlés par le franchiseur.

2 - L'efficacité, c'est la force d'un mode
strictement vérifiée par le franchiseur.

3 - La popularité, c'est la confirmation de la durée dans le temps et dans l'espace. Le franchiseur ne s'est pas endormi sur l'originalité de sa formule. Il en a vérifié, maintenu et développé l'efficacité. Il ne s'est pas laissé détourner de ses objectifs corporatifs; sa vigilance n'a pas été diluée dans l'extension de son modèle. Il a franchi le seuil de développement à partir duquel le modèle génère sa propre expansion. Il n'est plus nécessaire de démontrer son originalité ou son efficacité. La durée et l'espace de sa

Communication et organisation, 2 | 2012 
présence font, de ces deux points, des postulats à partir desquels le franchisé se convainc lui-même de l'intérêt qu'il retirera de son acquisition. Néanmoins cela ne veut pas dire qu'un «jeune » franchiseur mettant plutôt l'accent sur son originalité ne puisse pas représenter certains avantages comme des territoires potentiellement plus intéressants et une attention plus grande envers les besoins de chacun des franchisés (Croft et Whittemore, 1988).

\section{Ce qui est payé} consommation en spécifiant que l'offre précédait la demande pour le modèle faisant l'objet de la franchise. L'acheteur (le franchisé) identifie un besoin personnel (celui de faire fructifier son argent et/ou son temps) et non un besoin « environnemental». Ce travail a été fait par le franchiseur qui l'a incorporé dans les critères de séduction de son produit-modèle. est inportant de signaler que cette relation vendeur-acheteur, entre le franchiseur et le franchisé, dépasse le contexte d'un simple achat limité à la séquence d'acquisition, de réception et de jouissance d'un bien ou d'un service. Car la contrepartie de ces droits obtenus par «l'acheteur ", c'est le «prix à payer ». Or ce prix ne s'exprime pas en terme unique et final. Il s'agit plutôt d'un engagement financier à long terme dont la durée sera parallèle à celle de la jouissance du bien acheté, c'est-à-dire des avantages offerts par le statut de franchisé. qu'elle l'est aussi pour en assurer la rentabilité. L'échange commercial entre le franchiseur et le franchisé ne saurait être soumis aux lois et aux attentes d'une «vente finale ». Il ressemble plutôt au contrat de leasing ou location-bail avec deux grandes différences.

La première, c'est qu'il est impossible de prévoir la durée du contrat en fonction d'une usure calculée ou d'une péremption naturelle du produit (comme pour l'automobile ou l'ordinateur) permettant d'en distribuer le coût selon un calendrier précis. On devrait, au contraire, prévoir une augmentation de la valeur du produit au fur et à mesure de son implantation et de son expansion. Le franchisé serait ainsi amené à payer plus cher la jouissance d'un produit en fonction de sa valorisation progressive. Mais ce dernier pourra contrer cette démarche en se réclamant de droits acquis ou même de participation à la valeur ajoutée... à laquelle il contribue par sa présence et sa performance au sein du réseau.

Cette attente d'une valeur croissante de la franchise explique la deuxième différence : il est illusoire de fixer, une fois pour toutes, les conditions de l'échange (prix contre bénéfices). Car ces aspects sont influencés par le degré d'atteinte des objectifs du franchisé (la rentabilité de son investissement en temps et/ou argent) et l'évolution des trois critères de séduction offerts par le modèle du franchiseur. Même si la contribution du franchisé est fixée par contrat pour un terme plus ou moins long, le franchiseur doit tenir compte de la marge de manœuvre dont celui-ci dispose pour moduler son adhésion aux pratiques qui régissent le calcul et le versement de cette contribution. Il en découle une sorte de négociation permanente entre la satisfaction du franchisé et celle du 
franchiseur, dans la mesure où le premier pourra contribuer plus ou moins spontanément aux revenus du deuxième.

51 On devine déjà que cette relation entre le degré de satisfaction perçu par le franchisé et le prix qu'il est prêt à mettre pour obtenir cette satisfaction exigera de la part du franchiseur une stratégie de communication lui permettant non seulement de réaliser, chez le franchisé, les conditions de son modèle, mais aussi de suivre la perception de ce dernier face à ce même modèle.

\section{La stratégie de communication}

52 Le franchiseur doit donc mettre en place une stratégie de communication qui tienne compte des échanges d'informations permettant, à la fois, de réaliser son type de franchisage et d'orienter la perception des franchisés par rapport à l'évolution de ce modèle. La création et le fonctionnement de réseaux adéquats de communication peuvent être considérés comme l'un des services offerts par le franchiseur ou comme l'un des instruments permettant de réaliser les services autres que la communication. Dans tous les cas, ces réseaux semblent relever de l'initiative du franchiseur comme gardien et garant du modèle de franchise ${ }^{2}$.

Il est difficile de trouver dans les cadres théoriques généraux les éléments d'une typologie correspondant aux caractéristiques particulières d'un réseau de franchise. Pour faciliter le travail du gestionnaire, essayons d'en proposer une qui serait basée sur les éléments échangés entre le franchiseur et les franchisés, tels que décrits plus haut. Comme ces éléments existent rarement à l'état pur, il s'agit souvent d'un élément initial auquel les autres viennent progressivement se greffer sous la pression des événements ou la tension naturelle des relations franchiseur-franchisé.

Pour la bannière, il s'agit avant tout d'informer les franchisés sur les campagnes publicitaires projetées afin qu'ils commandent une quantité suffisante de produits ou qu'ils détiennent les ressources nécessaires pour offrir le service annoncé. On peut ici parler d'une communication verticale uni-directionnelle dont les composantes sont entièrement sous le contrôle du franchiseur. C'est lui qui décide du moment et du contenu de la communication. Cela se fait habituellement sous forme de communiqués ou de réunions périodiques. Dans ce dernier cas, on s'assure d'un feedback technique portant sur la bonne compréhension du message. Car le communiqué suppose une certaine routine avec des attentes réciproques parfaitement synchronisées.

Il y a aussi, pour le franchiseur, le besoin de contrôler l'utilisation de la bannière. S'il n'est pas dans un type de franchisage de diffusion-exploitation, il ne peut se permettre d'homogénéiser le cadre dans lequel la bannière est utilisée. Mais il est important que les principaux arguments assurant le pouvoir de séduction de la bannière soient respectés. L'originalité et l'efficacité de la bannière sont associées, dans les interventions promotionnelles, à certains éléments comme le service, la garantie ou les prix. Comme il ne peut intervenir directement dans la réalisation de ces éléments par chacun des franchisés, le franchiseur cherchera à les motiver plutôt qu'à les censurer. Il organisera des rencontres au cours desquelles il valorisera les comportements " constructifs » afin de favoriser l'incitation par l'exemple. Il créera ce que Deal et Kennedy (1982) appellent des "héros relatifs» dont les comportements sont ponctuellement signalés afin de 
développer une "méta-culture organisationnelle» liant les points forts des cultures locales des franchisés.

Les actions «culturisantes» du franchiseur sont fortement dépendantes de l'empathie existant entre les gestionnaires du franchiseur et ceux des franchisés. Cela se fera d'autant plus facilement s'il existe un dénominateur commun entre les partenaires, autre que le contrat de franchise. C'est le cas du franchiseur dont la bannière est associée à un « héros absolu » (Deal et Kennedy, 1982). Fondateur du réseau, il peut lui avoir donné son nom ou y être lié par une présence « charismatique » sur la place publique. Il devient un point de référence professionnel ou commercial dont on se plaît à imiter les comportements et soutenir les opinions.

\section{La dynamique de changement}

La description de la stratégie de communication d'une bannière annonce déjà une certaine tendance vers une modification du type de franchise. Pour assurer un approvisionnement adéquat des franchisés en période de promotion collective, le franchiseur en vient à se donner un mécanisme d'achat de produits dont il pourra confirmer le coût de revient à ses partenaires. Une fois en place, ce mécanisme génère son propre développement. La bannière se transforme progressivement en centrale d'achat. De « culturelles ", les communications deviennent opératoires. Il faut établir des procédures de commandes, de vérification, de livraison et de facturation. Celles-ci ne peuvent rester aléatoires. On établit des normes de fonctionnement qui ont des répercussions sur l'ensemble des activités du franchisé.

marge de manœuvre du franchisé se trouve réduite par les procédures de commande qui influencent son calendrier commercial ainsi que la répartition des tâches et des responsabilités au sein de son personnel. Quoi et quand commander? De proactive, ses opérations de commande deviennent réactives. L'initiative glisse de la périphérie (les franchisés) au centre (le franchiseur). Pour rentabiliser ses propres opérations d'achat, d'entreposage et de livraison, le centre va tout naturellement souhaiter une extension de son champ d'activité. Qui va établir la liste des produits dont l'achat est contrôlé par le franchiseur? Comment le franchiseur va-t-il financer ce nouveau service : en conservant une partie des économies de volume ou en allant chercher un pourcentage des chiffres de vente des franchisés? Pour réaliser une telle ponction en aval ou en amont de son intervention, il va devoir augmenter son propre personnel. Celui-ci cherchera à son tour à justifier son existence par un contrôle plus serré des franchisés... toujours au nom de l'efficacité de l'ensemble.

59 C'est ainsi que le franchisage de diffusion "modèle pur " glisse vers le franchisage de diffusion-exploitation. Le contrôle des achats entraîne celui du merchandising afin d'assurer une circulation adéquate des produits générant des économies de volume. Et la facturation modèle les procédures de gestion et de vente aux consommateurs. Les relevés de caisse et les inventaires doivent être harmonisés afin d'accélérer le traitement des données comptables et les transferts d'argent impliqués.

Cette évolution peut se réaliser en cours de fonctionnement. Mais il peut aussi faire partie de la réflexion menant à l'élaboration d'un projet de franchisage.

Même si le modèle initial a déjà officiellement intégré le franchisage de diffusionexploitation, sa réalisation contient toujours un certain pourcentage de frustrations pour 
le franchisé qui voit modifier ses habitudes de fonctionnement (dans le cas d'un changement de bannière) ou réduire ses rêves d'« intrapreneurship " (dans le cas d'un nouveau commerce). Et la dynamique interne d'un réseau ne saurait s'en tenir au cadre d'opérations décrit dans le contrat d'origine. Comme le contexte évolue, les besoins se modifient. L'apparition de nouvelles activités commerciales justifie l'extension du modèle; la part d'initiative réservée par le centre ne saurait diminuer sans remettre en question le leadership qui a justifié, au départ, l'adhésion du franchisé.

\section{Les agents et les bénéficiaires} communications sur une base individuelle entre chaque franchisé et chaque responsable des secteurs du centre. Les problèmes doivent demeurer ponctuels; ils ne sont que des accidents de parcours dans un cheminement qui ne peut être remis en question sans risquer d'avoir à reconnaître l'existence de tensions incompatibles avec l'image de séduction offerte par le modèle de franchise. D'ailleurs ces responsables des secteurs du centre subissent, comme tous les cadres intermédiaires, la tentation bureaucratique qui consiste à monopoliser l'information et, surtout, à éviter que ces «accidents de parcours » ne parviennent à l'attention de leurs supérieurs. Ils ont tendance à former une barrière entre les propriétaires de l'organisme franchiseur et ceux des franchises, coupant ainsi les liens organiques qui avaient donné naissance au modèle de franchise encore véhiculé par le discours officiel de l'ensemble.

Bien que cela ait été récupéré et institutionnalisé par certains franchiseurs sous le nom de «Franchise Advisory Councils» (FAC) (Justis, 1985), la création, par la base, d'une association de franchisés est l'un des symptômes les plus clairs d'une telle déstabilisation des relations franchiseur-franchisés. Ces derniers se sentent aliénés par rapport au centre qui ne répond plus à leurs attentes...ou à leurs appels. Le plus intéressant, c'est que ces appels portent la plupart du temps sur un retour à l'esprit de la bannière initiale. On juge que l'expansion du réseau et le gonflage du centre qui s'ensuit, a détruit les relations privilégiées qui unissaient le franchiseur aux franchisés. On se sent « succursalisé » alors qu'on voulait être «associé». Les griefs portent alors sur des accrocs à la pureté du 
modèle. Car un franchisé ne saurait contester le modèle sans remettre en question la sagesse de sa décision de joindre le réseau. Le centre peut donc tabler sur cette volonté de ne pas se livrer à une auto-critique indirecte ou encore de ne pas détruire l'efficacité publique du modèle auquel le franchisé est associé dans l'esprit de sa propre clientèle.

Le franchisé qui refuse l'extension du modèle vit une relation schizophrénique avec le franchiseur. Il voudrait refuser certaines des pratiques imposées par le centre. Mais la stratégie de développement de ce dernier interdit tout "opting out». Il ne peut que rejeter l'ensemble. Même s'il est prêt à passer par-dessus les obstacles légaux inscrits dans la plupart des contrats de franchise (Lockerby, 1985), il s'est emprisonné lui-même dans une bannière sur laquelle il a modelé ses activités commerciales. Il a aliéné la personnalité de son entreprise au profit de celle du franchiseur. Il ne peut que reculer devant les effets négatifs d'un divorce au sortir duquel il ne peut retrouver un fonctionnement autonome depuis longtemps atrophié par les communications normatives du franchiseur.

On atteint donc un équilibre apparent derrière lequel le franchiseur peut constamment augmenter sa part d'initiative sans tenir compte de la « grogne » des franchisés qu'il sait prisonnier de leur décision initiale d'adhérer au réseau.

\section{Les causes de déstabilisation}

68 L'équilibre précaire maintenu entre les attentes des franchisés et les pratiques du franchiseur peut être remis en question de différentes façons.

Il y a les causes externes reliées à l'évolution du contexte socio-économique. Cela peut être l'apparition d'un réseau concurrent (de succursales ou de franchises) misant sur une bannière plus séduisante et une promotion plus agressive. Par ailleurs les consommateurs peuvent modifier leurs habitudes, délaisser un produit ou une manière de se le procurer (passant, par exemple, du service à la table au service à l'auto ou vice-versa) et ainsi réduire la valeur de ce qui était l'argument de séduction du franchiseur. Ou le législateur peut redéfinir les réglementations touchant les pratiques commerciales ou l'exercice d'une profession et ainsi réduire l'originalité ou la popularité d'une franchise basée sur certains éléments qu'elle n'a plus le droit d'utiliser.

Les tensions inhérentes au système de franchise se trouvent libérées par l'annulation du chantage du franchiseur à l'exclusion du réseau. Si le bilan du franchisé est négatif, il n'a plus aucune raison de craindre la perte d'une bannière qu'il croit être en train de causer sa perte. Le franchiseur est donc condamné à réaliser la croissance de son réseau afin d'occuper une telle proportion de son champ économique qu'il pourrait apparaître plus dangereux, pour le franchisé mécontent, de s'en séparer que d'y rester associé même en période de bilan négatif.

71 Cette croissance du réseau peut entraîner une contraction des territoires réservés aux franchisés. Par exemple, la prise de contrôle ou l'intégration d'un réseau concurrent implique une redistribution des territoires entre les anciens et les nouveaux franchisés. Ce réajustement des droits des franchisés a des répercussions encore plus déstabilisantes lorsque le franchiseur se réserve la propriété de certains nouveaux points de vente, devenant ainsi un concurrent direct des franchisés occupant des territoires voisins. Car il existe une distance minimale en-deçà de laquelle le franchisé commence à se sentir menacé par la présence d'un autre franchisé. Le franchiseur pourra essayer de faire 
comprendre, relevés démographiques en main, que l'évolution de l'occupation du territoire lui permet de maintenir le bassin de clientèle potentielle. Le franchisé demeure focalisé sur ce qu'il perçoit comme la proximité agressive d'un autre franchisé. Il perd alors son « innocence corporative » en passant au crible de cette nouvelle concurrence toutes les politiques proposées par le franchiseur. Car, désormais, il ne se perçoit plus comme étant le seul bénéficiaire d'un avantage partagé par le concurrent.

\section{Les changements catalyseurs}

\section{changement dans les pratiques du réseau peut servir de catalyseur à l'éclatement} organisationnel.

un franchiseur doit apporter beaucoup d'attention au d'implantation informatique. Entrainant des changements importants (ou du moins perçus comme importants par le franchisé) dans leurs pratiques locales et dans celles du réseau, de tels projets forcent les partenaires à remettre en question leur rôle et leur intérêt dans l'ensemble. Ces nouvelles pratiques vont-elles favoriser le contrôle du centre ou l'autonomie de la périphérie ? Même si la première réponse s'inspire plus des préjugés individuels que d'une analyse poussée du projet annoncé, le franchiseur doit prendre le temps de tenir compte de ces préjugés avant même de tenter de décrire les implications techniques de son projet.

Or les gestionnaires du franchiseur vivent toujours la conviction d'être la locomotive qui tire le train de franchisés. Ceux-ci n'auront, encore une fois, qu'à constater, dans quelques mois, la validité des options prises pour eux. Mais ils oublient que les changements entraînés par un projet informatique touchent à l'«intimité » des entreprises franchisées. Ils redéfinissent les tâches et les rôles des propriétaires, de leurs gérants et de leurs employés. Il ne s'agit plus d'une opération passagère de marketing ou d'une réorganisation ponctuelle de quelques procédures de commande ou d'enregistrement de clients. C'est une démonstration à long terme et un rappel permanent des capacités d'intervention $d u$ franchiseur dans les opérations des franchisés.

\section{Pour réussir une implantation bureautique}

Il est extrêmement important de retrouver «l'esprit » original de la franchise afin de comprendre les paramètres conceptuels dans lesquels les franchisés vont intégrer le projet informatique. Si la culture de la bannière a mis l'accent sur l'importance d'un propriétaire "présent à sa clientèle ", il faut que les agents de changement respectent, dans leur stratégie d'implantation autant que dans leur discours, le rythme d'appréhension des franchisés face à cette nouvelle technologie. Même si on peut tabler sur les vertus de séduction du "techno-discours » ambiant, il n'est pas certain que les franchisés en saisissent toutes les implications organisationnelles. Il ne faudrait pas se contenter d'une acceptation (ou d'une « soumission ») passagère qui deviendrait d'autant plus dangereuse qu'elle serait justifiée par une ignorance, entretenue ou non, des effets à court et long termes de l'informatisation sur les pouvoirs et expertises dont la distribution assure la cohésion de chacune des entreprises. Il importe non seulement 
d'informer les franchisés sur les objectifs du projet, mais aussi de leur offrir un soutien « convivial » au point de vue technique et organisationnel.

Si la franchise est associée nominalement ou même historiquement à un « héros absolu » qui a justifié l'adhésion d'un bon nombre de franchisés, c'est lui qui doit assumer le leadership de l'implantation. Sinon, cette transformation majeure du vécu du franchisé sera perçue comme une déviation de l'esprit initial de la bannière parce qu'elle sera « imposée » par ces «bureaucrates-parasites » du franchiseur. On risque alors le rejet ou le boycottage pratique du projet sous prétexte qu'il s'agit d'une nouvelle «fantaisie » de «technocrates» en mal d'étendre l'influence de leur activité professionnelle et de leur pouvoir au sein du réseau. La participation active du "héros absolu» permettra de recentrer le débat sur les objectifs de l'ensemble et assurera aux franchisés la possibilité de recourir à l'imitation du modèle qu'il représente durant la période de turbulence organisationnelle suivant toute implantation informatique.

En l'absence d'un tel « héros absolu », les agents de changement auraient avantage à créer des « héros relatifs » qui pourraient jouer une partie du rôle de ce " héros absolu ». Cela se ferait en réalisant l'implantation chez deux ou trois franchisés dont les succès espérés seraient valorisés par les différents instruments de culture organisationnelle (Anand, 1987). On créerait ainsi des médiateurs entre les franchisés et les "bureaucrates » du franchiseur et on fournirait aux premiers des modèles avec lesquels ils auraient une meilleure complicité.

78 Le recours à ces " héros relatifs " pourrait même être utile dans les cas où le " héros absolu » maintient un rôle actif dans le changement proposé. Ils pourraient multiplier la valeur de ses interventions en misant sur la crédibilité que leur donnerait, auprès des autres franchisés, une association plus étroite avec ce dernier dans le cadre du projet d'implantation informatique. Sans compter que, dans un cas comme dans l'autre, une telle opération permettrait d'évaluer et d'ajuster le système proposé auprès de franchisés choisis pour leurs attitudes "positives" envers l'informatique et les initiatives du franchiseur!

\section{En conclusion}

Les gestionnaires d'un réseau de franchises doivent tenir compte de l'évolution des perceptions qu'entretiennent les franchisés quant aux facteurs de séduction du modèle impliqué. Quels sont les éléments qui assurent leur adhésion? Correspondent-ils à ceux qui alimentent la planification du franchiseur? Ce dernier est-il en train de renforcer le modèle "franchise de diffusion-exploitation" alors que le succès lui-même de la «bannière » ne semble aucunement justifier le besoin, l'effort et le coût de modifier les opérations? L'efficacité des réseaux de communication sera jugée en fonction de la rapidité avec laquelle ils indiqueront au franchiseur tout changement dans la perception des franchisés. Par ailleurs les mêmes réseaux devront fournir aux franchisés des explications sur les changements qu'ils peuvent percevoir comme des tentatives de déviance par rapport au modèle auquel ils ont adhéré (ou croient avoir adhéré).

Un réseau de franchises doit offrir aux partenaires des lieux de négociation constante entre les attentes des uns et des autres. Si on ignore volontairement les tensions inhérentes à cette dualité, on risque d'en voir réapparaitre les symptômes dans l'acceptation ou le rejet des vertus opératoires des projets proposés par le franchiseur. On 
rencontre alors des résistances ou même des blocages qui pourraient faire dévier ces projets de leurs objectifs de départ.

81 C'est pourquoi il est important de se donner les moyens de régler les dissonances culturelles (visions des modèles) avant de s'attacher à démontrer l'utilité et la faisabilité techniques de ces projets. Et cela s'applique plus particulièrement aux projets d'implantation informatique qui interviennent directement aussi bien dans les pratiques locales des franchisés que dans celles qui gèrent leurs relations commerciales et financières avec le franchiseur.

\section{BIBLIOGRAPHIE}

Anand, P., "Introducing Franchisees to Relinquish Control : An Attribution Analysis », Journal of Marketing Research, vol. 24 n² 2, mai 1987, pp. 215-221.

Andrews, E. L., "So Who Owns Us This Week?», Venture, vol. 9 n 10, octobre 1987. pp. 20-21.

Bracker, J. S. et Pearson, J. N., " The Impact of Franchising on the Financial Performance of Small Firms » , Journal of the Academy of Marketing Science, vol $14 \mathrm{n}^{\circ}$ 4, hiver 1988, pp. 10-17.

Croft, N. L. et Whittemore, M., « Finding the Right Franchise », Nation's business, vol 76 n² 2, février 1988, pp. 53-60.

Deal, T. E. et Kennedy, A. A., Corporate Cultures : the Rites and Rituals of Corporate Life, Reading, Addi son-Wesley, 1982.

Edens, F. N., Self, D. R. et al., «Franchisors Describe the Ideal Franchise », Journal of Small Business, vol $14 \mathrm{n}^{\circ}$ 3, juillet 1976, pp. 39-47.

Edwards, P., « How to Slip Safely into a Franchise », Rydge's, vol 60 n 2, février 1987, pp. 60-69. Jones, M. et Boughton, N., Phillips, P., «The New Moonlighters », Successful Executive, vol. $1 \mathrm{n}^{\circ} 5$, octobre 1986, pp. 20-30.

Justis, R., « Have you hugged your Franchisee Today? " Nation's Business, vol 73 n 2, février 1985, pp. 46-49.

Lee, L. W., « Franchising and Interbrand Competition », Southern Economic Journal, vol $51 \mathrm{n}^{\circ} 1$, juillet 1984, pp. 219-234.

Lockerby, M., "Franchise Termination Restrictions : A Guide for Practitioners and Policy Makers », Antitrust Bulletin, vol. 30 n $^{\circ}$ 4, hiver 1985, pp. 791-871.

Stern, A.L., " New Payoff from Old Brand Names », Dun's Business Month, vol $125 \mathrm{n}^{\circ}$ 4, avril 1985, pp. 42-44.

Tauber, E. M., « Brand Franchise Extension : New Product Benefits from Existing Brand Names », Business Horizons, vol. $24 \mathrm{n}^{\circ}$ 2, mars-avril 1981, pp. 36-41. 


\section{NOTES}

1. Voir l'histoire du eu Adventureland Video Inc., l'un des plus gros franchiseur américains en ce domaine, dont les membres insatisfaits ont décidé de créer leur propre coopérative d'achat Indépendante de ce dernier (Andrews, 1987).

2. D'après une étude réalisée en 1976, les franchiseurs ne semblaient pas (du moins à cette époque !) accorder beaucoup d'importance aux habiletés de communication des candidats à la franchise qu'ils souhaitaient plutôt «dynamiques, optimistes et coopératifs » (Edens et Self. 1976).

\section{RÉSUMÉS}

L'informatisation interne verticale des activités de gestion intervient aussi bien dans les pratiques locales des franchisés que dans celles qui gèrent leurs relations commerciales et financières avec le franchiseur. Il est important de retrouver «l'esprit » original de la franchise afin de comprendre les paramètres conceptuels dans lesquels les franchisés vont intéger le projet informatique. En prenant conscience des implications communicationnelles des différents types de franchisage de reproduction ou de "diffusion"sur les structures organisationnelles. le franchiseur doit se donner les moyens de régler les dissonances culturelles (visions des modèles) avant de s'attacher à démonter l'utilité et la faisabilité techniques de son projet. 